\title{
Caracterización de recién nacidos a término con deshidratación hipernatrémica
}

\section{Characterization of full term newborns with hypernatremic dehydration}

\author{
Del Castillo C. Gabriel ${ }^{\mathrm{a}, \mathrm{b}}$, Suares A. Diana ${ }^{\mathrm{a}}$, Granja A. María ${ }^{\mathrm{a}}$, \\ Oviedo E. Bibiana ${ }^{a}$, Urbano U. Jhuranny ${ }^{\mathrm{a}}$, Cabrera B. Nancy ${ }^{\mathrm{a}, \mathrm{c}}$
}

aFundación Hospital Infantil Los Ángeles, San Juan de Pasto, Colombia

bUniversidad de Nariño, San Juan de Pasto, Colombia

'Fundación Universitaria Cátolica del Sur, San Juan de Pasto, Colombia

Recibido: 27 de agosto de 2019; Aceptado: 5 de agosto de 2020

¿Qué se sabe del tema que trata este estudio?

La deshidratación hipernatrémica neonatal, una condición en aumento como causa de ingreso a las unidades de cuidado intensivo neonatal, es sospechada cuando hay pérdida anormal de peso $>10 \%$, irritabilidad, hipertermia, ictericia y puede llevar a falla renal o hemorragia del sistema nervioso central.
¿Qué aporta este estudio a lo ya conocido?

Este estudio identificó variables clínicas que pueden contribuir a que los profesionales de salud identifiquen de forma oportuna factores de riesgo, tomen medidas preventivas, de tratamiento, y se eviten complicaciones y desenlaces fatales.

\section{Resumen}

La deshidratación hipernatrémica neonatal es una condición grave y su incidencia se ha incrementado en los últimos años, repercutiendo en complicaciones que llevan a la hospitalización del recién nacido. Objetivo: Describir las características clínicas y de laboratorio de recién nacidos a término con diagnostico de deshidratación hipernatremica. Pacientes y Método: Estudio observacional descriptivo de recién nacidos a término que se hospitalizaron por deshidratación hipernatrémica entre los años 2014 y 2016. Se incluyeron recién nacidos a término mayores de 37 semanas con signos clínicos de deshidratación (mucosas secas, fontanela deprimida, llanto sin lágrimas, signos de pliegue cutáneo) y/o pérdida excesiva de peso mayor de 7\% y sodio sérico mayor a 145 $\mathrm{mEq} / \mathrm{L}$. Se registraron variables sociodemográficas y bioquímicas para su análisis. Resultados: Se incluyeron 43 neonatos. El 60,5\% de sus madres fueron primigestantes, el 90\% de los neonatos recibieron lactancia materna exclusiva, las madres reportaron problemas en la lactancia materna en el $76,7 \%$. La pérdida de peso al ingreso con respecto al peso de nacimiento fue de $15,3 \%$ en pro-
Palabras clave: Deshidratación; Recién Nacido; Hipernatremia; Lactancia Materna 
medio. El 83,3\% contaba con seguro de salud público. 65,1\% presentó signos clínicos de deshidratación al ingreso y $83,5 \%$ signos neurológicos transitorios. El promedio de sodio fue de $155 \mathrm{mEq} / \mathrm{L}$ al ingreso. El descenso de sodio en las primeras 24 horas de manejo fue $7,74 \mathrm{mEq} / \mathrm{L}(0,32 \mathrm{mEq} / \mathrm{L}$ por hora). La corrección de la hipernatremia fue en el 55,8\% por vía oral y la estancia hospitalaria de 4 días en promedio. Conclusiones: Los problemas de alimentación se presentaron en un (76\%) madres primigestantes en un $(88,4 \%)$. El 90,6\% de esta población administraban lactancia materna exclusiva, resultados que pueden contribuir para alertar al profesional de la salud a identificar de forma oportuna, signos de alarma y un control precoz posterior al alta del puerperio y a la toma de medidas preventivas.
Keywords: Dehydration; Infant; Hypernatremia; Breast Feeding

\section{Introducción}

La deshidratación hipernatrémica neonatal (DHN) es una condición caracterizada por la pérdida anormal de peso, signos clínicos de deshidratación y una concentración sérica de sodio $>145 \mathrm{mEq} / \mathrm{L}$ en el menor de 28 días $^{1,2}$. La pérdida fisiológica de peso en los recién nacidos a término, no debe superar entre el 7\%-10\% en los primeros días de vida ${ }^{1,3,4}$, una pérdida mayor es un signo de alarma para evaluar una posible DHN; puesto que existe una relación directa entre la pérdida de peso y deshidratación hipernatrémica ${ }^{2}$.

Esta incidencia parece incrementar según los reportes de la literatura a nivel mundial teniendo en cuenta que entre el 1-1,8\% de las admisiones neonatales son causadas por esta patología ${ }^{5}$.

Esta condición clínica relativamente común es motivo de preocupación debido a que el potencial de morbilidad es significativo. La verdadera incidencia del problema no se ha determinado claramente debido a que los estudios realizados no incluyen a los recién nacidos prematuros, ni pacientes con problemas como paladar hendido, síndrome hipotónico y trisomía 21 entre otros ${ }^{6,7}$. La DHN se ha asociado a morbilidades tales como: hemorragia del sistema nervioso central (SNC), trombosis venosa cerebral, coagulopatía, apnea, falla renal, ictericia y secuelas neurológicas a largo plazo $^{2,7}$.

El manejo de esta condición clínica en recién nacidos sigue siendo controvertida porque los neonatos tienen signos clínicos de deshidratación menos evidentes y son más susceptibles a la deshidratación por la inmadurez renal, la distribución de agua corporal y porque las necesidades de agua y sodio son diferentes en niños mayores ${ }^{8}$. Cuando los recién nacidos no son tratados adecuadamente, pueden tener graves complicaciones y secuelas neurológicas a largo plazo. El objetivo de este estudio es describir las características clínicas y de laboratorio de recién nacidos a término con diagnóstico de deshidratación hipernatremica en una Unidad Neonatal de un hospital infantil de referencia. 


\section{Pacientes y Método}

Estudio observacional, descriptivo-retrospectivo de recién nacidos que ingresaron al Hospital Infantil Los Ángeles de Pasto. Colombia, durante el período de enero de 2014 a diciembre de 2016. Para los efectos de este estudio se incluyeron recién nacidos a término hospitalizados con signos clínicos de deshidratación y/o pérdida de peso mayor al 7\% y un sodio sérico mayor a $145 \mathrm{mEq} / \mathrm{L}$. Fueron recolectados los datos registrados en las historias clínicas teniendo en cuenta la descripción de variables sociodemográficas del neonato: edad en días, género, seguro de salud; variables clínicas del neonato: problemas de alimentación, ictericia, fiebre, peso al nacer, peso al ingreso, tipo de alimentación, signos de deshidratación, signos neurológicos, conducta de soporte enteral y parenteral, ictericia, fototerapia y días de estancia; variables de laboratorio y radiológicas: hallazgos radiológicos, descenso del sodio en las primeras $24 \mathrm{~h}$, sodio, calcio, cloro, creatinina, potasio y glicemia al ingreso; variables clínicas de la madre: edad, paridad, vía de parto, semanas de gestación. Se diseñó un instrumento de recolección de información en Excel versión 2014.

Se incluyeron recién nacidos a término mayores de 37 semanas que tuvieran signos clínicos de deshidratación (mucosas secas, fontanela deprimida, llanto sin lágrimas, signos de pliegue cutáneo) y/o pérdida excesiva de peso mayor de 7\% y sodio sérico mayor a $145 \mathrm{mEq} / \mathrm{L}$. Se excluyeron a aquellos pacientes que desarrollaron hipernatremia durante la hospitalización, malformaciones congénitas mayores, pacientes que no tuvieran todos los datos en el instrumento e información incompleta en la historia clínica. Los sesgos de información se controlaron realizando la revisión de historias clínicas por 6 de los investigadores, quienes estandarizaron las definiciones de las variables,

\begin{tabular}{l}
$\begin{array}{l}\text { Tabla 1. Características sociodemográficas y clínicas de las } \\
\text { madres de los recién nacidos a término con deshidratación } \\
\text { hipernatrémica }\end{array}$ \\
\begin{tabular}{llll}
\hline Característica & Variable & Categoría & $43(\%)$ \\
\hline $\begin{array}{l}\text { Características de } \\
\text { la madre }\end{array}$ & $\begin{array}{l}\text { Edad de la materna } \\
(\mathrm{M} \pm \mathrm{DE} \pm)\end{array}$ & \multicolumn{2}{c}{$25,4(6,95)$} \\
& Paridad & $\begin{array}{l}1 \text { a } 2 \text { hijo } \\
\text { 3 a } 4 \text { hijos }\end{array}$ & $38(88,4)$ \\
& & $\begin{array}{l}\text { Más de } 4 \\
\text { hijos }\end{array}$ & $1(9,3)$ \\
& & Vaginal & $24(55,8)$ \\
& Vía de parto & Cesárea & $19(44,2)$ \\
& & & $39,2(0,87)$ \\
& Edad gestacional en \\
semanas (M DE \pm$)$
\end{tabular} \\
\hline
\end{tabular}

crearon un proceso organizado para la revisión de las historias clínicas para no pasar por alto detalles o información valiosa y realizaron una prueba piloto en la que registraron y analizaron los primeros 10 casos de dicho evento.

\section{Análisis de la información}

Los programas utilizados para la recolección, procesamiento y análisis fueron: software SPSS ${ }^{\circledR}$, versión 21 (IBM Corp. Released 2012. IBM SPSS Statistics for Windows, versión 21.0. Armonk, NY: IBM Corp.) Presentación de resultados en Microsoft Word.

Se realizó descripción de todas las variables de la población de estudio, teniendo en cuenta la naturaleza de las mismas; para las variables cualitativas se utilizaron frecuencias absolutas y relativas y para variables cuantitativas se empleó el promedio, desviación estándar y media.

Se respetaron los principios éticos fundamentales contemplados en la declaración de Helsinki. El proyecto fue avalado por el comité de ética del Hospital Infantil los Ángeles de la Ciudad de Pasto, Colombia, el 27 de noviembre de 2014. Este estudio se clasificó como "Riesgo mínimo" de acuerdo con el artículo 11 de la Resolución 8430 de 1993, Ministerio de Salud de Colombia.

\section{Resultados}

Durante el período analizado para esta investigación, el total de ingresos a la unidad neonatal fue de 505 recién nacidos a término, quienes ingresaron a la institución pediátrica entre enero de 2014 y diciembre de 2016; de esta población, 43 presentaron signos clínicos de deshidratación o pérdida de peso mayor al $7 \%$. El 51\% fueron mujeres, con promedio de edad de 6 días a su ingreso; el $83,7 \%$ se encontraba afiliado al sector público del sistema de seguridad en salud.

El promedio de edad gestacional en semanas correspondió a 39 semanas de gestación, el 88,4\% de las madres tenían un hijo y un promedio de edad de 25 años, el 55\% de los pacientes nacieron por vía vaginal (tabla 1).

La media de peso al nacer se encontraba en 3.238 $\mathrm{g}(\mathrm{DE} \pm 474 \mathrm{~g})$, el promedio del peso al ingreso fue de $2.736 \mathrm{~g}$ y el promedio de pérdida de peso fue de $15,3 \%$ (DE $\pm 7,88 \%$ ) (tabla 2). De los niños analizados, el $90,6 \%$ fue alimentado exclusivamente con leche materna, las madres reportaron problemas en su alimentación en un $76,7 \%$; se encontró que el $65 \%$ de esta población presentó signos clínicos de deshidratación (oliguria, fontanela deprimida, signos de pliegue, mucosas secas) y el $83 \%$ presentó algún signo neurológico (irritabilidad, letargia, hipertonía, somnolencia). Se 
Deshidratación hipernatrémica - G. Del Castillo C. et al

Tabla 2. Características sociodemográficas y clínicas de los recién nacidos a término con deshidratación hipernatrémica

\begin{tabular}{|c|c|c|c|}
\hline Característica & Variable & Categoría & $43(\%)$ \\
\hline \multirow{15}{*}{$\begin{array}{l}\text { Características sociodemográficas } \\
\text { y clínicas del neonato }\end{array}$} & Peso al nacer $\mathrm{g} \quad(\mathrm{M} \pm \mathrm{DE} \pm)$ & $3,238(474)$ & \\
\hline & Peso al ingreso g $\quad(M \pm D E \pm)$ & $2,736(452)$ & \\
\hline & Pérdida de peso g $(M \pm D E \pm)$ & $15,3(7,88)$ & \\
\hline & Edad - días $\quad(\mathrm{M} \pm \mathrm{DE} \pm)$ & $6,3(4,87)$ & \\
\hline & Género & $\begin{array}{l}\text { Masculino } \\
\text { Femenino }\end{array}$ & $\begin{array}{l}21(48,8) \\
22(51,2)\end{array}$ \\
\hline & Seguro de salud & $\begin{array}{l}\text { Privado } \\
\text { Público }\end{array}$ & $\begin{array}{r}7(16,3) \\
36(83,7)\end{array}$ \\
\hline & Tipo de alimentación & $\begin{array}{l}\text { Lactancia materna exclusiva } \\
\text { Fórmula artificial } \\
\text { Mixta (lactancia materna, fórmula, aromática) }\end{array}$ & $\begin{array}{rr}39 & (90,6) \\
1 & (2,3) \\
3 & (6,9)\end{array}$ \\
\hline & Problemas de alimentación & & $33(76,7)$ \\
\hline & Fiebre & & $17(39,5)$ \\
\hline & Ictericia & & $36(83,7)$ \\
\hline & Fototerapia & & $23(53,4$ \\
\hline & Signos de deshidratación & & $28(65,1)$ \\
\hline & Síntomas neurológicos & & $36(83,7)$ \\
\hline & Corrección de hipernatremia & $\begin{array}{l}\text { Oral } \\
\text { Intravenosa } \\
\text { Oral e intravenosa (mixta) }\end{array}$ & $\begin{array}{r}24(55,8) \\
13(30,2) \\
6(13,9)\end{array}$ \\
\hline & Días de hospitalización $(\mathrm{M} \pm \mathrm{DE} \pm$ ) & $4,62(4,59)$ & \\
\hline
\end{tabular}

encontró que la corrección de la hipernatremia (reposición de agua libre) se realizó en su mayor parte por vía oral en un $55,8 \%$, el $30,3 \%$ por vía intravenosa y $13,9 \%$ fue mixta (oral /intravenoso) (tabla 2).

En las variables clínicas, el 39\% presentó fiebre cuantificada y el $83,7 \%$ ictericia en el examen físico; a 6 pacientes $(14 \%)$ se le realizó estudios de neuroimagen así: 3 pacientes $(6,9 \%)$ ecografía transfontanelar, 2 pacientes $(4,6 \%)$ tomografía cerebral y 1 paciente $(2,3 \%)$ tomografía cerebral y ecografía transfontanelar; encontrando en un caso hemorragia intraventricular grado 1 . El promedio de sodio al ingreso fue de $155 \mathrm{mEq} / \mathrm{L}$ con una (DE $\pm 8 \mathrm{mEq} / \mathrm{L})$. El descenso de sodio en las primeras $24 \mathrm{~h}$ de manejo fue $7,74 \mathrm{mEq} / \mathrm{L}$ ( $0,32 \mathrm{mEq} / \mathrm{L}$ por hora) (figura 1$)$. Se evaluaron pruebas de función renal: BUN con un promedio de $26,0 \mathrm{mg} / \mathrm{dL}$ y creatinina $0,92 \mathrm{mg} / \mathrm{dL}$, el promedio del potasio fue de 4,3 (DE \pm $0,6)$, y del cloro 117 ( $\mathrm{DE} \pm 19$ ), la glicemia tuvo un promedio de $70 \mathrm{mg} / \mathrm{dL}(\mathrm{DE} \pm 31 \mathrm{mg} / \mathrm{dl})$ (tabla 3$)$.

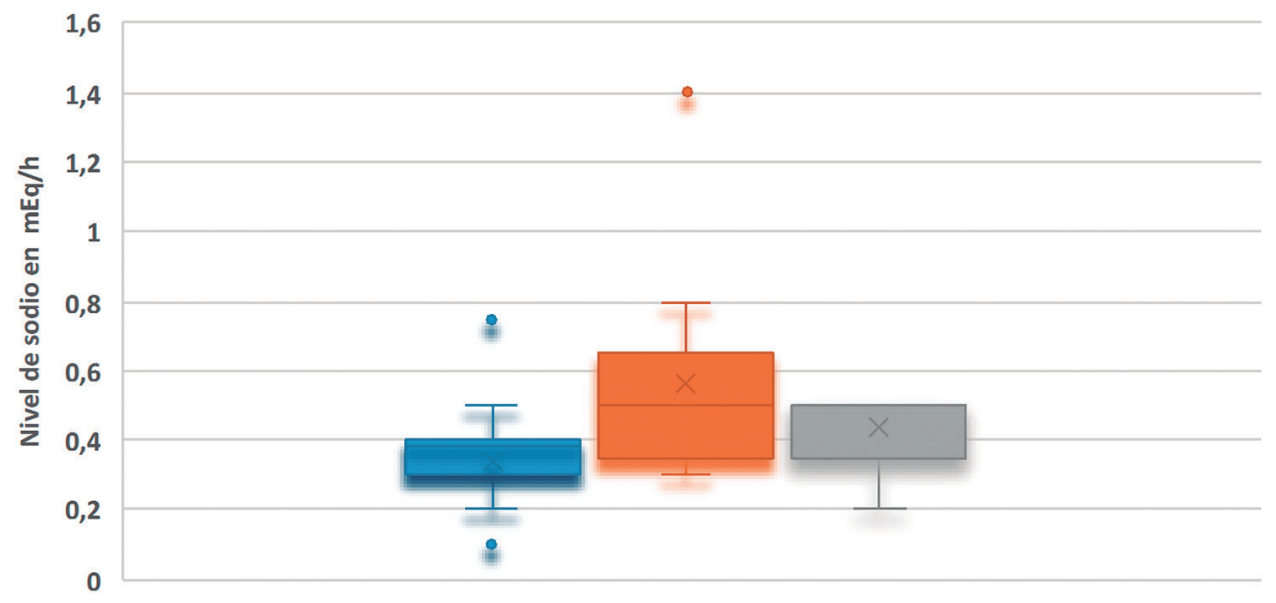

Vía oral $\square$ Vía intravenosa $\square$ Mixta (vía oral / vía intravenoso)

Figura 1. Disminución de sodio en 24 h por hora según tipo de soporte. 
Tabla 3. Características de laboratorio de los recién nacidos a término con deshidratación hipernatrémica

\begin{tabular}{lll}
\hline Característica & Variable & $(\mathrm{M} \pm \mathrm{DE})$ \\
\hline Característica de laboratorio & Descenso del sodio en las primeras $24 \mathrm{~h}$ & $7,7(8,84)$ \\
& Glicemia $\quad \mathrm{mg} / \mathrm{dl}$ & $70(31,1)$ \\
& Creatinina $\mathrm{mg} / \mathrm{dl}$ & $0,92(0,30)$ \\
& Sodio $\quad \mathrm{mEq} / \mathrm{l}$ & $155(8,06)$ \\
& Potasio $\quad \mathrm{mEq} / \mathrm{l}$ & $4,3(0,61)$ \\
& Cloro $\quad \mathrm{mEq} / \mathrm{l}$ & $117(19,1)$ \\
& Nitrógeno ureico en sangre mg/dl & $26(23,9)$ \\
\hline
\end{tabular}

\section{Discusión}

La deshidratación hipernatrémica (DHN) es una condición de hospitalización común en los recién nacidos a término en los primeros días de vida y ha venido ganando importancia por su comportamiento ascendente reportado en la literatura clínica9.

La incidencia de la DHN es variable; se encuentran reportes de 2,5 por cada 10.000 nacidos vivos en la serie de Oddie et al. ${ }^{2}$, Manganaro et al. reportan una incidencia de $7.7 \%$ entre los niños alimentados exclusivamente con leche materna ${ }^{10}$. Uras et al. reportan una incidencia de $5,6 \%{ }^{11}$. Moritz et al. reportan una incidencia tan baja como $1,9 \%$ en niños hospitalizados a término ${ }^{1}$. En este estudio, la incidencia fue de 8,5\% de los ingresos a la unidad neonatal durante el período estudiado; se observa un porcentaje notablemente mayor al reportado en la literatura, se considera que es necesario documentar este hallazgo y plantear estrategias de abordaje de un problema aún no resuelto.

Similar a los ejemplos de publicaciones de otros países, esta situación es más frecuentemente en estratos socioeconómico bajos, representado en este estudio por los afiliados al sector público de la salud. También influye la alimentación con lactancia materna exclusiva y problemas de alimentación, posiblemente este último relacionado con una galactopoyesis inefectiva en las primeras horas manifestado por problemas de alimentación referidos por las madres, sumado a técnicas inadecuadas tanto de posición como de agarre durante la lactancia. Además coincide la presentación con mayor frecuencia en madres primíparas sin experiencia en el cuidado del recién nacido, falencias en la de educación sobre la lactancia materna, el alta temprana o problemas como pezones planos e invertidos no detectados durante el control prenatal ${ }^{6}$.

El rango de sodio sérico normal se encuentra entre $135 \mathrm{mEq} / \mathrm{l}$ a $145 \mathrm{mEq} / \mathrm{L}$, por lo que toda elevación del sodio sérico por encima de $145 \mathrm{mEq} / \mathrm{l}$, se considera hipernatremia ${ }^{1,11}$. En este estudio se definió hipernatremia como un sodio $>145 \mathrm{mEq} / \mathrm{l}$. Algunas publicaciones definen como hipernatremia neonatal valores superiores de $150 \mathrm{mEq} / \mathrm{L}^{12,13}$.

La gran mayoría de la población estudiada presentó al menos uno de los signos clínicos de deshidratación como: oliguria, fontanela deprimida, mucosas secas, signos de pliegue; estos signos son signos inespecíficos y se presentan en una gran variedad de enfermedades en el período neonatal. Uno de los signos clínicos más específicos es la disminución de peso, siendo un hallazgo importante para establecer el diagnóstico como lo soportan otros estudios revisados ${ }^{1,10,14}$. Sin embargo, en la literatura de los últimos años no es claro el porcentaje de pérdida de peso definida como normal en los primeros días de vida de los recién nacidos a término que son alimentados exclusivamente con leche materna, no hay datos fisiológicos confiables. Algunos artículos estiman que la pérdida de peso en la primera semana de vida corresponde al $10 \%{ }^{1,3,4}$, refiriendo que una pérdida superior al 10\% aumenta 47 veces el riesgo de desarrollar deshidratación hipernatrémica; otros autores refieren que una perdida mayor al $7 \%$ en los primeros tres o cuatro días de vida es un signo de alarma y hay que buscar su causa ${ }^{10,15,16}$. Los pacientes de este estudio tuvieron en promedio una pérdida de peso de $15.6 \%$ con respecto al peso de nacimiento, Tabla 2. Con un promedio de edad al ingreso de 6,3 días, estos resultados se correlacionan con la literatura ${ }^{3,16}$.

Según la literatura, la meta terapéutica en el manejo de la DHN es corregir el choque hipovolémico y realizar un descenso de la osmolaridad sérica con una disminución del sodio que no supere $0,5 \mathrm{mEq} / \mathrm{l}$ por hora ${ }^{14}$. Para este estudio, la reducción fue de $0,32 \mathrm{mEq} / \mathrm{l}$ por hora en promedio en toda la población, con tratamiento vía oral la reducción de sodio fue de $0,3 \mathrm{mEq} / \mathrm{hora}$, con vía intravenosa fue de $0,5 \mathrm{mEq} / \mathrm{hora}$, mixto (vía oral y vía intravenosa) fue de $0,35 \mathrm{mEq} /$ hora, según lo recomendado (figura 1 ). 
La estrategia más utilizada para la corrección del sodio en esta población de estudio fue la vía oral: es fácil de manejar, reduce los costos en líquidos endovenosos, requiere menos frecuencia en el control de los niveles de sodio y facilita su uso en centros de salud de menor complejidad ${ }^{17}$. Es importante mencionar que el descenso de los niveles de sodio fue más lento cuando se utilizó la vía oral que con la vía endovenosa (figura 1). Según Erdemir y colaboradores, se elige esta vía, si es bien tolerada y no hay contraindicaciones ${ }^{17}$. Cabe destacar que la DHN puede conducir a la presentación de secuelas neurológicas a largo plazo $^{1}$, en esta investigación se encontró que la mayoría de pacientes presentaron al ingreso algún signo neurológico transitorio, pero no se realizó seguimiento a largo plazo después de la hospitalización. En quienes se realizó neuroimágenes, como hallazgo se documentó un caso de hemorragia intraventricular grado 1 y el resto se reportaron como normales.

La literatura sugiere que entre los signos clínicos que se manifiestan en pacientes con DHN está ubicada en los primeros lugares la ictericia ${ }^{14,16}$. En este estudio la ictericia fue el signos más frecuente; un $83,7 \%$ de los recién nacidos presentó ictericia en el examen físico y requirió tratamiento con fototerapia a diferencia del estudio realizado por Saxena et al. quienes encontraron menor porcentaje de niños con ictericia ${ }^{18}$.

Respecto a las características encontradas en las madres de este estudio, se encontró una edad promedio de 25 años, el 88,4\% tuvieron un hijo, hay varios estudios que han relacionado la falla en la alimentación como un factor importante asociado a la DHN en recién nacidos a término aparentemente $\operatorname{sanos}^{19}$; en el estudio de Boskabadi, en Irán, reportaron que el 50\% de los casos de DHN de su serie está asociado a este problema $^{20}$. El estudio canadiense de Livingstone detectó que el $70 \%$ de las madres con bebés deshidratados y falla de alimentación eran primigestantes ${ }^{21}$. El estudio de Caglar y Ozzer también concluye que 44\% de las madres de bebés con DHN y problemas de alimentación eran primíparas ${ }^{15}$. Los estudios sugieren que hay mayor riesgo de DHN en los hijos de madres primigestantes ${ }^{3,22-24}$ como se evidencia en el presente estudio. Ninguno de los pacientes incluidos en esta revisión falleció durante su estadía hospitalaria.

Este tipo de estudio únicamente permite explorar algunas características de la población que ingresó al hospital. Considerando su diseño se debe tener en cuenta que existen algunas limitaciones como la selección inicial de las historias clínicas a partir del código CIE 10, que pudo generar la no inclusión de historias clínicas de pacientes con DHN. Además, otra limitación fue la recolección de datos tomados del registro en las historias clínicas que no siempre presentaban todas las variables definidas.

\section{Conclusiones}

En el presente estudio se identificó importantes variables clínicas y sociodemográficas que deben alertar al profesional de la salud a identificar al recién nacido con deshidratación hipernatremica, entre las que se destacan: madres primigestantes, perdida significativa de peso del lactante, problemas de alimentación y características clínicas que incluyen manifestaciones neurológicas como signos de deshidratación.

Un importante hallazgo fue la alta frecuencia de los problemas de alimentación, valdría la pena explorar en un estudio posterior sus causas y su verdadero valor, para poder tomar las medidas preventivas adecuadas, como por ejemplo asegurar una adecuada técnica de lactancia materna antes del alta, además de un seguimiento ambulatorio con enfermería o pediatría oportuno especialmente a madres y/o recién nacidos en los que se encuentre algún factor de riesgo.

\section{Responsabilidades Éticas}

Protección de personas y animales: Los autores declaran que los procedimientos seguidos se conformaron a las normas éticas del comité de experimentación humana responsable y de acuerdo con la Asociación Médica Mundial y la Declaración de Helsinki.

Confidencialidad de los datos: Los autores declaran que han seguido los protocolos de su centro de trabajo sobre la publicación de datos de pacientes.

Derecho a la privacidad y consentimiento informado: Los autores han obtenido el consentimiento informado de los pacientes y/o sujetos referidos en el artículo. Este documento obra en poder del autor de correspondencia.

\section{Conflicto de intereses}

Los autores declaran no tener conflicto de intereses. 


\section{Referencias}

1. Moritz ML. Breastfeeding-Associated Hypernatremia: Are We Missing the Diagnosis? Pediatrics [Internet]. 2005;116(3):e343-7. Available from: http://pediatrics.aappublications.org/cgi/ doi/10.1542/peds.2004-2647.

2. Oddie SJ, Craven V, Deakin K, Westman J, Scally A. Severe neonatal hypernatraemia: a population based study. Arch Dis Child Fetal Neonatal Ed [Internet]. 2013;98(5):F384-7. Available from: http://fn.bmj.com/content/98/5/ F384.long.

3. Karadeniz Bilgin, Leyla. Akcay, Fatima. Altinkaynak, Konca. Altindag H. Hypernatremia in Breastfed Newborns: a Review of 149 Cases. 2011;58(4):332-4.

4. Ghaleh A, Hosseini MB, Farhangmehr T. Evaluation of Dehydration and Hypernatremia in Neonates Admitted to Tabriz Children 's Hospital. 2009;31(2):2009.

5. Bischoff AR, Dornelles AD, Carvalho CG. Treatment of Hypernatremia in Breastfeeding Neonates: A Systematic Review. Biomed Hub. 2017;2(1):1-10

6. Lavagno C, Camozzi P, Renzi S, et al. Breastfeeding-Associated Hypernatremia: A Systematic Review of the Literature. J Hum Lact. 2016;32(1):67-74.

7. Ergenekon E, Unal S, Gücüyener K, et al. Hypernatremic dehydration in the newborn period and long-term follow up. Pediatr Int. 2007;49(1):19-23.

8. Ali US, Sengupta K, Andankar P, Saraf S, Chawla A, Deshpande S. Reversible renal medullary hyperechogenicity in neonatal hypernatremic dehydration. Pediatr Nephrol. 2004;19(9):1050-2.

9. Juliao J. Deshidratación hipernatrémica e hiperbilirrubinemia indirecta en el recién nacido a término. RevMedicaSanitas. 2009;12(4):34-42.
10. Manganaro R, Mamì C, Marrone T, Marseglia L, Gemelli M. Incidence of dehydration and hypernatremia in exclusively breast-fed infants. J Pediatr. 2001;139(5):673-5.

11. Uras N, Karadag A, Dogan G, Tonbul A, Tatli MM. Moderate hypernatremic dehydration in newborn infants: retrospective evaluation of 64 cases. J Matern Fetal Neonatal Med [Internet]. 2007;20(6):449-52. Available from: http://www.ncbi.nlm.nih.gov/ pubmed/17674254.

12. Koklu E, Gunes T, Ozturk MA, Kose M, Kurtoglu S, Yuksel F. A review of 116 cases of breastfeeding-associated hypernatremia in rural area of central Turkey. J Trop Pediatr. 2007;53(5):34750.

13. Peñalver Giner O, Gisbert Mestre J, Casero Soriano J, Bernal Ferrer A, Oltra Benavent M, Tomas Vila M. Deshidratación hipernatrémica asociada a lactancia materna. An Pediatr. 2004;61(4):340-3.

14. Bolat F, Oflaz MB, Güven AS, et al. What Is the Safe Approach for Neonatal Hypernatremic Dehydration? Pediatr Emerg Care [Internet]. 2013;29(7):808-13. Available from: http://content.wkhealth. com/linkback/openurl?sid=WKPTLP:land ingpage\&an $=00006565-201307000-00007$.

15. Çağlar MK, Özer I, Altugan FŞ. Risk factors for excess weight loss and hypernatremia in exclusively breastfed infants. Brazilian J Med Biol Res. 2006;39(4):539-44.

16. Barman H, Das BK, Duwarah SG. Acute kidney injury in hypernatremic dehydration in exclusively breastfed babies: don't ignore it! J Clin Neonatol. 2014;3(218):124-5.

17. Erdemir A, Kahramaner Z, Cosar H, et al. Comparison of oral and intravenous fluid therapy in newborns with hypernatremic dehydration. J Matern Fetal Neonatal Med [Internet]. 2014;27(5):491-4. Available from: http://www.ncbi.nlm.nih. gov/pubmed/23805991.

18. Saxena A, Kalra S, Shaw SC, et al. Correction of hypernatremic dehydration in neonates with supervised breastfeeding: A cross-sectional observational study. Med J Armed Forces India [Internet]. 2019;(xxxx). Available from: https://doi.org/10.1016/j. mjafi.2019.05.002.

19. Unal S, Arhan E, Kara N, Uncu N, Aliefendio?lu D. Breast-feeding-associated hypernatremia: Retrospective analysis of 169 term newborns. Pediatr Int [Internet]. 2008;50(1):29-34. Available from: http://doi.wiley.com/10.1111/ j.1442-200X.2007.02507.x [citado el 15 de junio de 2017].

20. Boskabadi H, Maamouri G, Ebrahimi $\mathrm{M}$, et al. Neonatal hypernatremia and dehydration in infants receiving inadequate breastfeeding. Vol. 19, Asia Pacific Journal of Clinical Nutrition. 2010. p. 301-7.

21. Livingstone VH, Willis CE, Abdel-Wareth LO, Thiessen P, Lockitch G. Neonatal hypernatremic dehydration associated with breast-feeding malnutrition: A retrospective survey. Cmaj. 2000;162(5):647-52.

22. A. Jonguitud-Aguilar, S. Calvillo-Robles, E. Ruiz-Martínez GO-L. Protocolo de manejo en deshidratación hipernatrémica neonatal. Perinatol Reprod Hum. 2015;29(2):65-9.

23. Morton J. The Clinical Usefulness of Breast Milk Sodium in the Assessment of Lactogenesis. Pediatrics. 1994;93(5).

24. NEIFERT M. Prevention of Breastfeeding Tragedies. Pediatr Clin North Am [Internet]. 2001;48(2):273-97. Available from: http://linkinghub.elsevier.com/ retrieve/pii/S0031395508700269. 